\title{
Energy Analyzer Attachments for the Scanning Electron Microscope
}

\section{A. Khursheed}

\section{Department of Electrical and Computer Engineering, National University of Singapore, Singapore.}

The Scanning Electron Microscope (SEM) is a high resolution imaging instrument, indispensable to research and development in Nano Science and Nano Technology. By integrating electron energy analyzers into its specimen chamber, it has the potential to become a powerful analytical tool that can map material science information on the Nano-Scale. This paper will review some selected aspects of research work aimed at developing electron energy analyzers attachments for the SEM.

One of the most important requirements for energy analyzer attachments in the SEM is that they must be small. Figure 1a is a schematic diagram illustrating the limited space around the specimen. The distance between the final lens lower pole-piece and the specimen, the working distance, usually ranges between 3 to $20 \mathrm{~mm}$, and for high resolution, it should be as small as possible. This means that the analyzer attachment height should typically be less than $20 \mathrm{~mm}$. Another important requirement for the analyzer is that it should have a wide angular range of collection and detection, since the scattered electrons from the specimen travel back in all directions. Ideally, the analyzer should have a $2 \pi$ radian azimuthal angle collection/detection range. The analyzer's ability to accept and detect electrons emitted over a wide range of angles is the main factor which limits its transport efficiency, which in turn, determines how short its data-acquisition times can be.

There are a variety of different possible applications for energy analyzers in the SEM, and they are best understood with respect to the part of the scattered electron energy spectrum that they are designed to capture. Figure 1b illustrates the three main categories of scattered electrons that are of interest: Secondary Electrons (SEs), in the low energy range (0 to $50 \mathrm{eV}$ ), Backscattered Electrons (BSEs), between $50 \mathrm{eV}$ and the primary beam energy (ranges up to $30 \mathrm{keV}$ ), and the Auger Electrons (AEs), between $0.1 \mathrm{keV}$ to $2.5 \mathrm{keV}$. Applications based upon the energy analysis and capture of low energy SEs include techniques such as quantitatively measuring voltage changes on the track of an IC, known as quantitative voltage contrast. This has historically been the main application driving development of energy analyzers for the SEM [1,2]. Recently however, other applications beyond voltage contrast are beginning to emerge. The SE energy spectrum has been used to map variations in the dopant level of semiconductor samples [3]. BSE energy analyzers were used to provide depth information about multilayer samples [4]. The possibility of carrying out Auger Electron spectroscopy in the SEM has been proposed, based upon cleaning a sample's surface by an ion flood gun, and then acquiring the AE spectrum through fast parallel energy detection [5]. A Transmission Electron Energy-Loss Spectrometer (EELS) attachment for the SEM has also been reported [6].

There seems to be some confusion in recent work on SE energy filtering about which figures of merit are relevant for SE energy analyzers. In a review written by J. Cazaux, he makes the incorrect statement that "the use of toroidal spectrometers is of limited interest because of their poor energy resolution in the energy range of interest" [7]. P. Kazemain et al, in the context of designing a SE energy analyzer for dopant concentration mapping, compare their results to unfiltered SE signals, which are mainly qualitative in nature, making no reference to the large body of work that had been previously reported on quantitative voltage contrast [3]. 


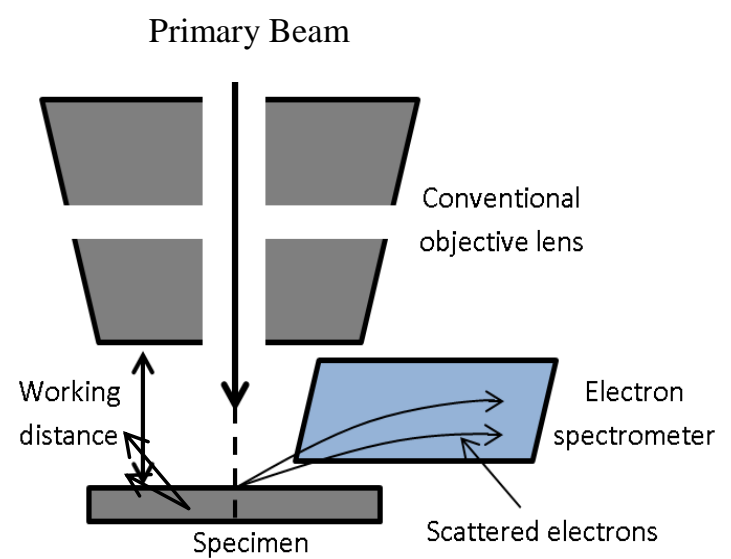

(a)

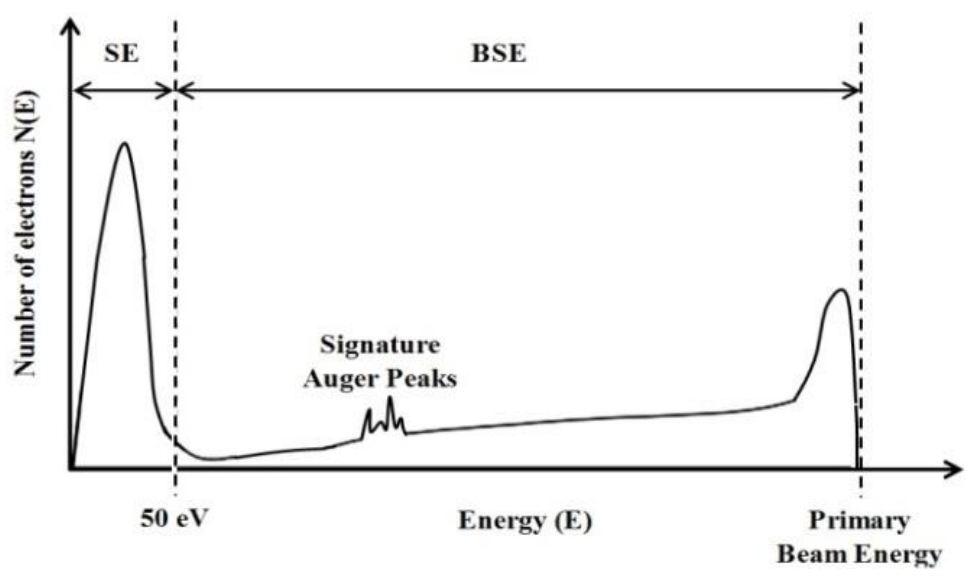

(b)

Figure 1. SEM layout. (a) Space for an analyzer attachment, (b) Scattered electron energy spectrum.

The following discussion will show that unlike the case for Auger Electron Spectroscopy (AES), it is not the energy resolution of the spectrometer that determines the voltage resolution of a SE analyzer, but signal to noise limits set by shot noise, moreover, this has been well documented and reported over twenty years ago in the context of voltage contrast analyzers for Electron Beam Testing [1,2].

A widely reported voltage contrast analyzer design used in the past is shown in Figure $2 \mathrm{a}$. This analyzer is of the Retarding Field type and was designed by Feuerbaum [8]. The analyzer has a height of only 10 $\mathrm{mm}$ and is fitted on to the specimen stage of the SEM. An extraction grid at a high potential $(<600 \mathrm{~V})$ above the specimen is used to minimize the effect of surface fields. Secondary electrons are accelerated up through the extraction grid and subsequently decelerated by the retarding field grid, whose potential is ramped in time. Secondary electrons that have enough initial energy to overcome the potential barrier created by the specimen-retarding gird voltage difference, $e\left(V_{R}-V_{S}\right)$, are then deflected through an exit grid located in the upper part of the analyzer and focused on to a SE detector. This analyzer has been simulated to have a transport efficiency of around $97 \%$ for SEs in the 0 to $10 \mathrm{eV}$ range [9]. The output signal of this analyzer, formed by ramping the retarding grid voltage in time, is an integrated form of the SE spectrum, which shifts in position as the specimen voltage changes. Since it is shifts in the output signal that are detected, it is important to understand that the voltage resolution of this analyzer is determined by shot noise, and not by the energy resolution of the spectrometer. This was experimentally confirmed and expressed in previous voltage contrast literature for retarding field analyzers by the wellknown Gopinath signal-to-noise formula [10].

Figure 3a shows a toroidal band-pass SE analyzer designed by Khursheed and Hoang [11, 12]. In this case, only secondary electrons within a narrow energy range are able to reach the detector, and the output signal is formed by ramping the analyzer deflector plate voltage (pass energy) in time, as shown in Figure 3b. The output signal in this case, directly represents the SE energy spectrum. The analyzer is rotationally symmetric and since it is small enough to be placed on the specimen stage $(<18 \mathrm{~mm}$ height), it can detect an azimuthal angular range up to around $300^{\circ}$, leaving around $60^{\circ}$ for the $\mathrm{SE}$ imaging detector. It should be noted that this toroidal SE analyzer has better focusing properties, greater transport efficiency, and a lower working distance than a previous SEM toroidal analyzer attachment reported for the SEM [4]. 


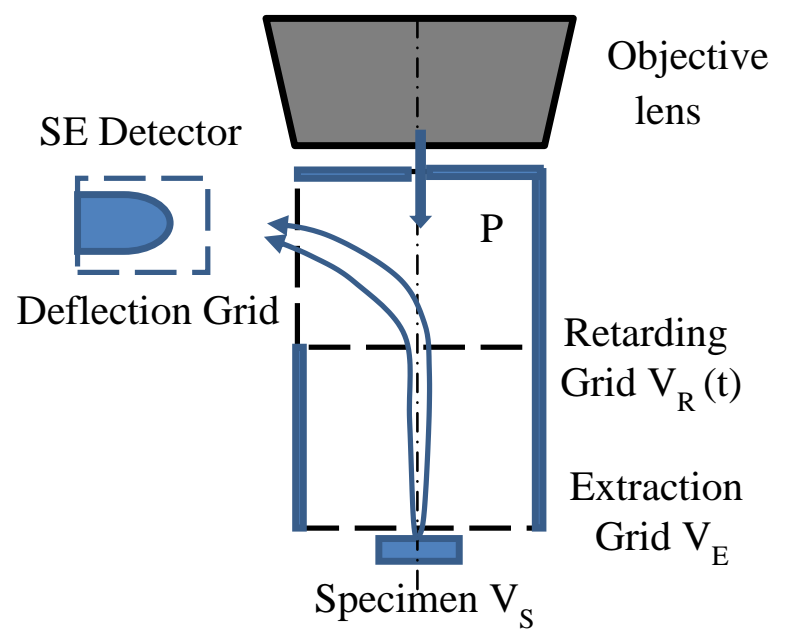

(a)

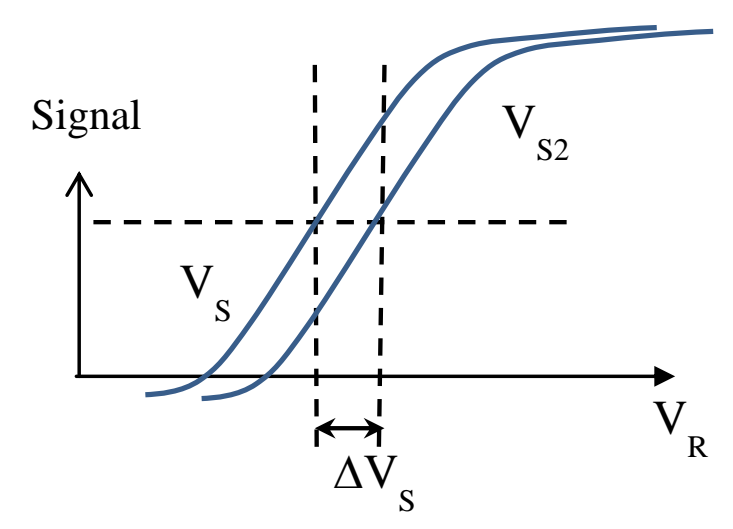

(b)

Figure 2. A Retarding Field SE analyzer example [8]. (a) Layout, (b) Output signal information.

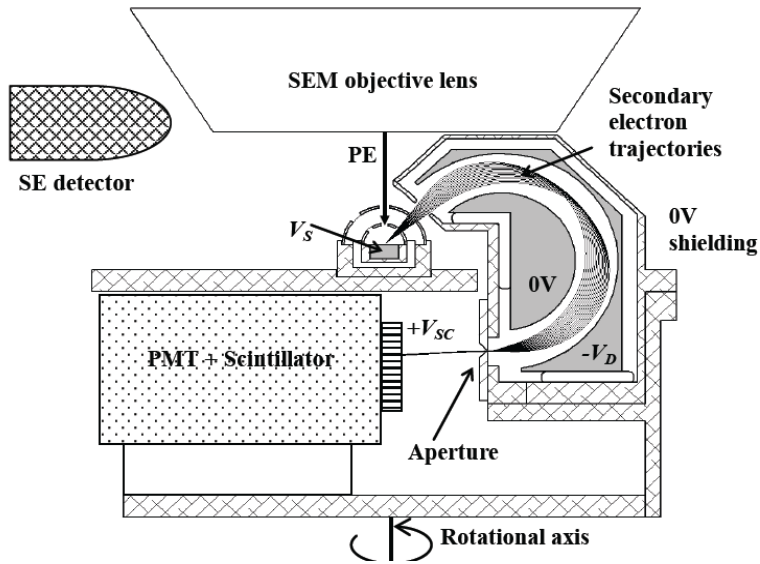

(a)

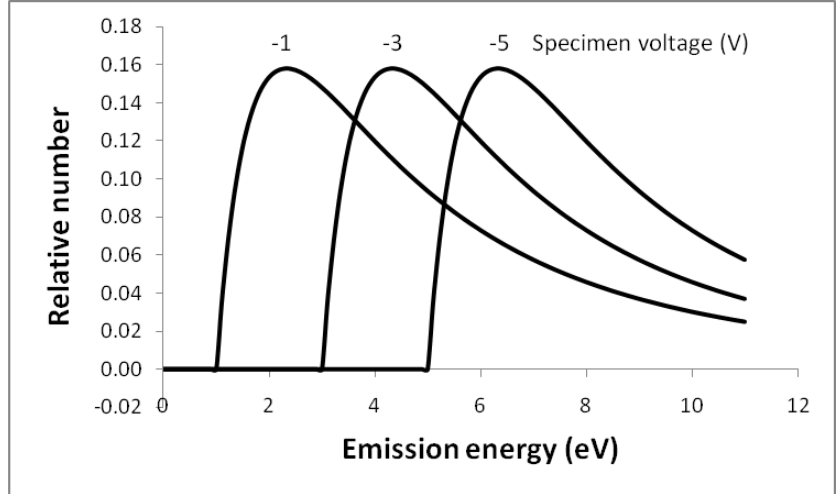

(b)

Figure 3. A bandpass analyzer design [11,12]. (a) Layou, (b) Output signal information.

Detailed studies comparing the signal-to-noise characteristics of retarding field analyzers with multichannel analyzers or bandpass analyzers were made in the past, and they concluded that obtaining the SE spectrum directly has over an order of magnitude better signal-to-noise characteristics [1]. This is because the retarding field signal is made from SEs that have a large range of energies, contributing mainly to the background noise, while changes to the actual signal only come from SEs having energies at or around the potential energy barrier. This is unlike the situation where the SE signal is obtained directly. In that case, changes at each energy (or energy channel) are monitored directly, information across a wide range of energies contributes to the signal, therefore, changes in the output signal position or shape are captured with greater sensitivity. Changes in a general parameter, such as the signal mean (or expectation value) can be monitored and related to changes in specimen potential. The effect of analyzer energy resolution here is to either broaden or sharpen the shape of the SE curve, but it does not limit the resolution to which changes in the signal's position or shape can be monitored.

Hoang et al recently reported a way to further enhance the signal-to-noise performance of the toroidal SE energy analyzer [13]. This technique is based upon creating an acceleration field at the entrance of 
the analyzer, which acts to pull in wider angle low energy secondaries into the analyzer. The acceleration field strength also increases the analyzer pass energy, effectively enlarging the detected energy bandwidth (and thereby increasing the output signal). The acceleration field is created by using two hemispherical caps between the specimen and analyzer entrance, and biasing both the specimen and inner cap to the same negative voltage, while leaving the outer cap voltage to be $0 \mathrm{~V}$, as shown in Figure 4. The figure shows $0.1 \mathrm{eV}$ secondary electron simulated trajectory paths plot by the Lorentz 2EM software [14] and experimental analyzer output signals taken from a bulk metal specimen for a range of negative specimen/inner cap voltages. The signal increases in height, by over an order of magnitude, and becomes more symmetric, enhancing its signal to noise characteristics.
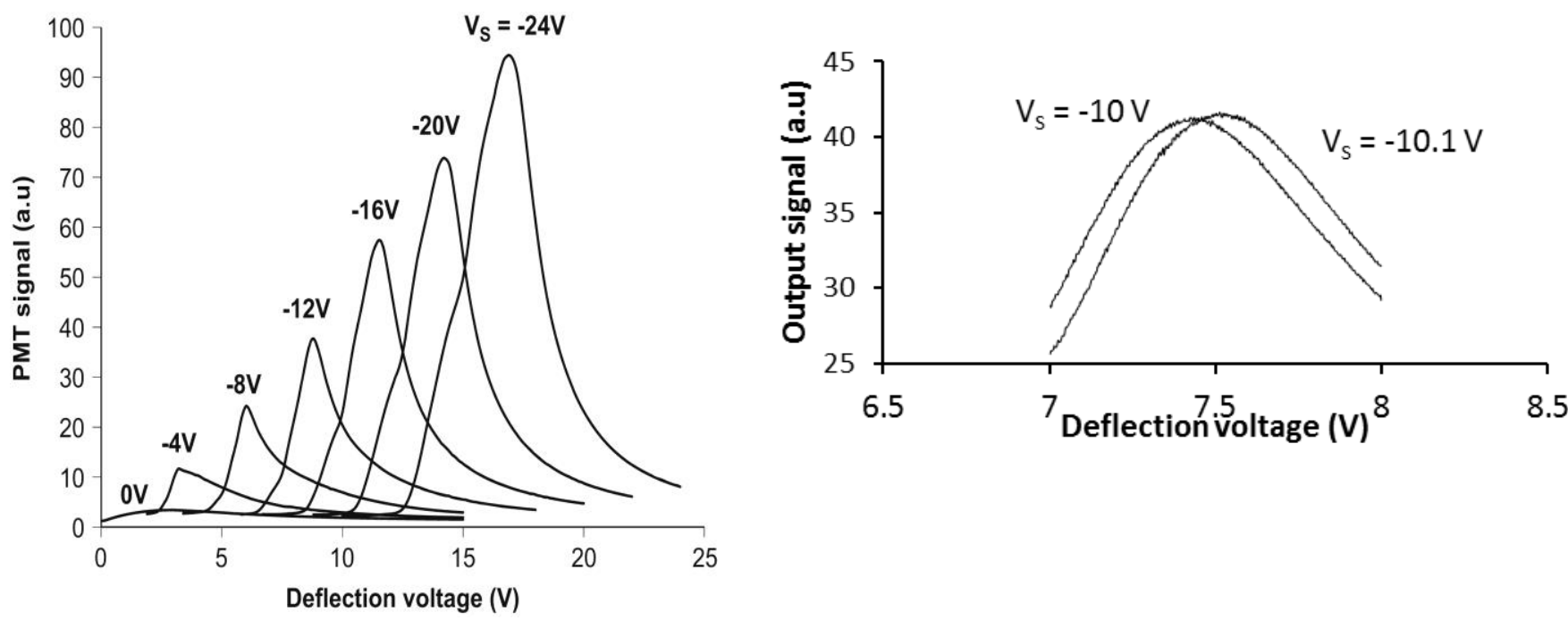

Figure 4. Enhancing the toroidal analyzer signal-to-noise characteristics through the creation of an acceleration field at the analyzer entrance

Figure 4 also shows how the output signal changes with a small change from -10 to $-10.1 \mathrm{~V}$ in the specimen/inner cap voltage, and by comparing the variation in the signal expectation value to that caused by noise, a signal to noise ratio of 2840 was obtained, see reference [13] for more details. If we assume that the shot noise sets the minimum measurable change in specimen potential, this translates into a minimum measurable specimen voltage change of around $32.5 \mu \mathrm{V}$. Of course, whether such a small change in specimen potential can be achieved in practice depends not only on shot noise, but other noise sources, such as power supply instabilities. However, in terms of the limit set by shot noise, it does represent one of the lowest ever reported. These results, as well as previous research work carried out in the subject of Electron Beam Testers, point towards band-pass or multi-channel SE energy analyzers being the better type of analyzers to use for applications such as dopant concentration mapping in the SEM. Research groups working in this area are apparently not aware of this. Kazemain et al not only used a retarding field analyzer for their PN junction dopant measurements, but their results were extracted from a very noisy portion of the output signal (S-curve) [3]. 
Another important aspect of SE energy analyzer design is the influence of surface fields and whether an extraction field is really required. To answer this question, a recent experiment using a modified version of the toroidal SE analyzer originally designed by Khursheed and Hoang was carried out in the presence of fringe fields between the specimen and analyzer entrance [15], as shown in Figure 5a. In this case, the specimen voltage $V_{S}$, is allowed to be more negative than the inner cap voltage, $V_{C l}$, which is set to a fixed voltage of $-10 \mathrm{~V}$, while the outer cap voltage, $V_{C 2}$, remains at $0 \mathrm{~V}$.

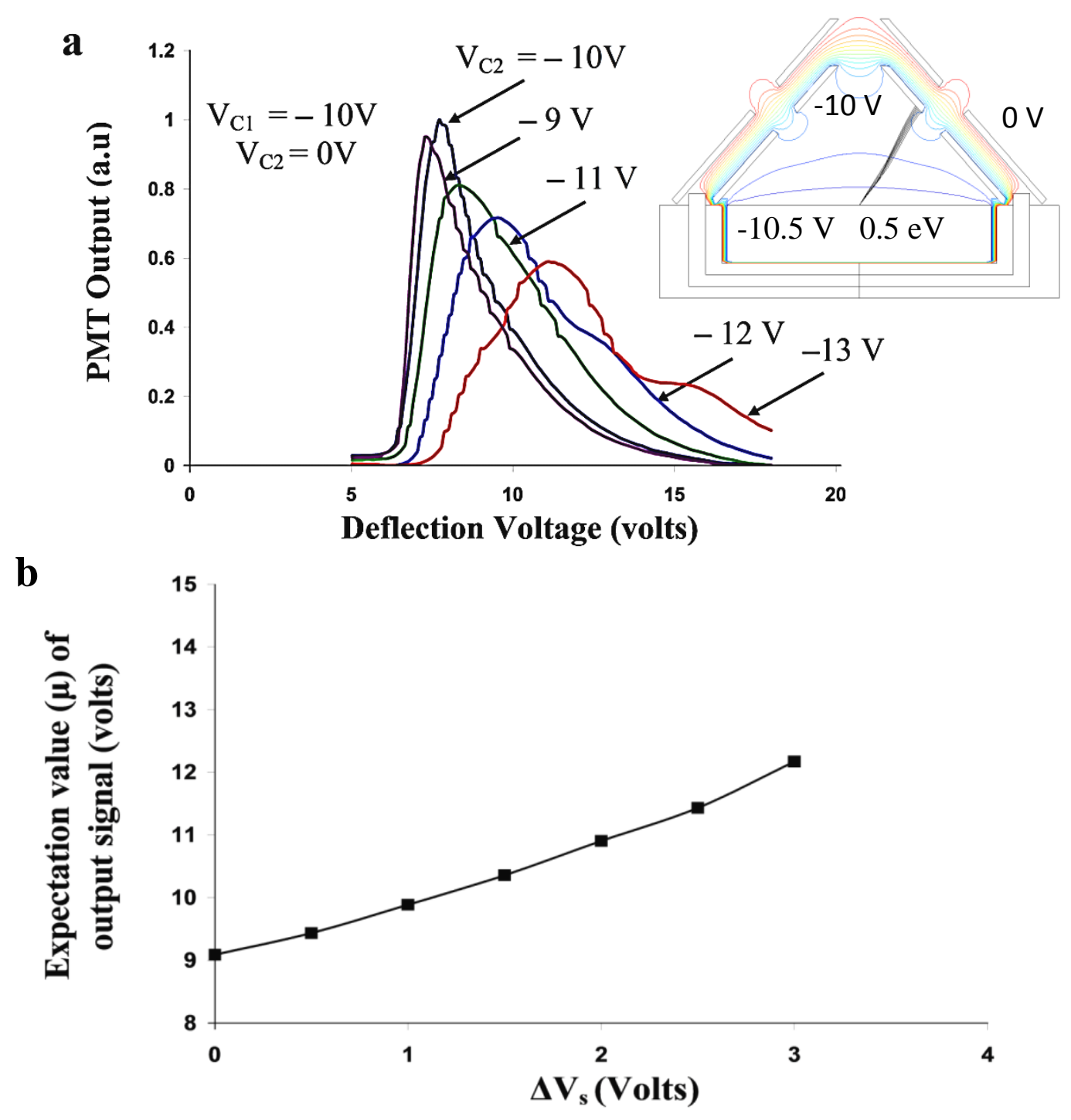

Figure 5. Experimental results from the toroidal SE analyser in the presence of fringe fields between the specimen and analyzer entrance (a) Experimental output signals, (b) Variation of the output signal expectation value.

The fringe fields created between the specimen and inner cap will significantly change the trajectory paths of secondary electrons, as illustrated in Figure $5 \mathrm{a}$, where the paths of $0.5 \mathrm{eV}$ secondary electrons over an angular range of $\pm 3^{\circ}$ are plotted for $V_{S}=-10.5 \mathrm{~V}$ and $V_{C l}=-10 \mathrm{~V}$ by the Lorentz 2EM software. In general, the effect of surface fields at the specimen will not be as severe, since surface fields are 
confined to a small region immediately above the specimen (typically tens of microns high), and do not usually extend up to the analyzer entrance, however, the fringe fields depicted in Figure 5a represent an extreme case: if the toroidal analyzer can measure specimen voltage changes in the presence of these fringe fields, they will also be able to measure specimen voltage changes in the presence of surface fields. Figure 5a indicates that the toroidal analyzer output signals are significantly different from the field-free region case depicted in Figure 4. They confirm the kind of simulations shown in Figure 5a, where low energy electrons no longer pass through the analyzer and therefore cause a drop in the output signal height as the strength of the fringe fields increase. However, despite this, the signal expectation value still monotonically increases as the specimen voltage becomes more negative, as shown in Figure 5b. Although not linear, once the nature of this dependence is taken into account, it is possible to correlate variations in the output signal expectation value to changes in the specimen voltage. Similar results have been obtained for measurements taken in the presence of surface fields [15]. The general conclusion is that since the toroidal SE analyzer can track specimen voltage changes in the presence of specimen fringe/surface fields, an extraction field is not required. [16]

\section{References:}

[1] A Khursheed in "Scanning Electron Microscope Optics and Spectrometers", (World Sci.) ch. 5.

[2] JTL Thong in "Electron Beam Testing Technology", (Plenum Press) ch. 5.

[3] P Kazemain et al, J. Appl. Phys. 100 (2006), p. 054901.

[4] E Rau et al, Rev. Sci. Instrum. 73 (2002), p. 227.

[5] MM El-Gomati, CG Walker and X Zha, Nucl. Instrum. Meth. A 645 (2011), p. 68.

[6] T Luo and A Khursheed, IEEE T. Device Mat. Res. 6 (2006), p. 182.

[7] J Cazaux, J. Electron Micros. 61 (2012), p. 277.

[8] HP Feuerbaum, Scan. Electron Micros. I (1979), p. 465.

[9] A Khursheed and AR Dinnis, Scanning 6 (1984), p. 85.

[10] A Gopanath, J. Phys. E. Sci. Instrum. 4 (1977), p. 334.

[11] A Khursheed and HQ Hoang, Ultramicroscopy 109 (2008), p. 104.

[12] HQ Hoang and A Khursheed, J. Vac. Sci. Technol. B 27 (2009), p. 3226.

[13] HQ Hoang, M Osterberg and A Khursheed, Ultramicroscopy 111 (2011), p. 1093.

[14] LORENTZ-2EM, Integrated Engineering Software Inc., Canada.

[15] A Srinivasan, PhD thesis, National University of Singapore, expected to be available in early 2015.

[16] The authors acknowledge funding under the Singapore Government MOE 2011-T2-2-108 grant. 